\section{Un trio de choc pour pallier la chimiorésistance des cancers c-MYC, PARP1, BIN 1}

Slovénie Pyndiah ${ }^{1,2}$, Daitoku Sakamuro ${ }^{1,3}$
${ }^{1}$ Molecular signaling program,

Stanley S. Scott cancer center, Louisiana state university, Health sciences center, New Orleans,

Louisiana 70112, États-Unis ;

${ }^{2}$ Institut de génétique moléculaire, cibles thérapeutiques, Inserm U940, 27, rue Juliette Dodu, 75010 Paris, France ;

${ }^{3}$ Department of biochemistry and molecular biology, GHSU cancer center, Georgia health sciences university, Augusta, Georgia 30912, États-Unis. slovenie.pyndiah@inserm.fr
> Vaincre la résistance des cellules cancéreuses à la chimiothérapie est devenu un vrai défi en oncologie clinique. Cette résistance peut survenir dès les premières cures de chimiothérapie, sans phase de sensibilité initiale, témoignant d'une résistance intrinsèque des cellules. En revanche, la résistance acquise se traduit par une progression tumorale survenant secondairement, après une phase de grande chimiosensibilité. Les mécanismes moléculaires impliqués dans ces phénomènes de résistance ont été très étudiés au cours de ces dernières décennies. La dérégulation de gènes codant pour des protéines membranaires, comme le gène multi drug resistance (MDR) qui code pour des pompes d'efflux rejetant le médicament à l'extérieur de la cellule, réduit l'efficacité thérapeutique des molécules anticancéreuses [1]. Au niveau du foie, le MDR joue un rôle physiologique dans la détoxication cellulaire. Dans les cellules cancéreuses ayant acquis une résistance, le MDR est alors activé. Cependant, d'autres mécanismes de résistance existent probablement. Ainsi, certaines tumeurs possèdent des altérations spécifiques du système de réparation des dommages à I'ADN les rendant vulnérables ou résistantes aux médicaments qui ont pour cible l'ADN. Par exemple, le système de réparation de I'ADN des cellules cancéreuses qui sont porteuses d'une mutation des gènes breast cancer 1 ou 2 (BRCAl ou 2) est défectueux, ce qui rend ces cellules extrêmement sensibles aux agents intercalants de I'ADN et aux inhibiteurs de la poly-ADP-ribose polymérase (PARP) [2, 3].
BIN1, un suppresseur de tumeur

Protéine participant à de multiples fonctions cellulaires, BINl (bridging integrator 1) intervient, entre autres, dans la réorganisation du cytosquelette, la polarité, le trafic cellulaire et dans la régulation de la transcription. Au moins dix isoformes ont été décrites, qui diffèrent par leur répartition tissulaire et leur localisation subcellulaire qui sont spécifiques. L'isoforme 9 de BINI (qui contient I'exon 13), majoritairement nucléaire, est présente dans pratiquement tous les types cellulaires [4]. Cette isoforme interagit avec $\mathrm{c}-\mathrm{MYC}$ et peut inhiber le pouvoir tumorigène des cellules malignes surexprimant C-MYC [5]. Le taux de BINI (toutes isofomes confondues) est diminué dans la majorité des tumeurs et des lignées cancéreuses humaines, et la réexpression de BINl (isoforme 9) dans ces cellules entraîne alors un déclin de la croissance cellulaire, voire la mort cellulaire programmée $[5,6]$. Ces observations suggèrent que BINl pourrait jouer le rôle d'un suppresseur de tumeur qui serait dérégulé dans divers cancers chez I'homme (Figure 1).

Dans un travail récent, nous montrons que $B I N 1$ peut aussi réguler la machinerie de réparation de I'ADN en inhibant PARPl (Poly [ADP-ribose] polymerase 1), une enzyme impliquée dans la réparation de I'ADN [7]. L'abondance cellulaire de BINl est corrélée positivement à la sensibilité aux composés chimiques à base de platine, comme le cisplatine, anticancéreux bien connus provoquant des dommages à l'ADN. En revanche, l'absence de BINl est associée à une importante résistance aux traitements à base de cisplatine. L'apport exogène de BINl dans des lignées cancéreuses promeut la sensibilité au traitement, indépendamment du statut de p53 [7]. Par ailleurs, dans des essais in vitro, les cellules développant spontanément des résistances au cisplatine ont un taux faible de BINl, et un apport exogène de cette molécule restaure une sensibilité au traitement [7]. Par conséquent, une déficience de BINl semble être associée à une progression de la résistance aux composés à base de platine.

\section{BIN1 inhibe PARP1, une enzyme clé dans la réparation des dommages à I'ADN}

Une analyse protéomique a permis d'identifier une interaction directe entre BINl et PARPl. PARPl est abondamment associée à la chromatine, ce qui lui permet de jouer son rôle de « gendarme » et de garantir la stabilité du génome. Lorsque PARPl détecte des lésions de I'ADN, elle s'active et catalyse la synthèse de polymères d'ADP-ribose (PAR) à partir de la nicotinamide adénine dinucléase $\left(N A D^{+}\right)$, utilisée comme précurseur. Les unités de PAR sont transférées à différentes protéines nucléaires cibles et PARPI constitue l'une des principales cibles. Cette production de PAR modifie localement la structure de la chromatine pour faciliter le recrutement des protéines impliquées dans la réparation de I'ADN cellulaire et fixer les dommages à I'ADN [8]. Des inhibiteurs chimiques de PARP augmentent l'efficacité des thérapies utilisant des molécules créant des dommages à I'ADN. Ce type de traitement est radical dans le cas où $B R C A l$ 
Cancer résistant à la chimiothérapie

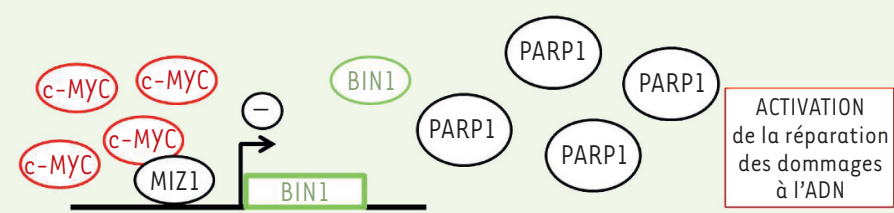

Cancer sensible à la chimiothérapie

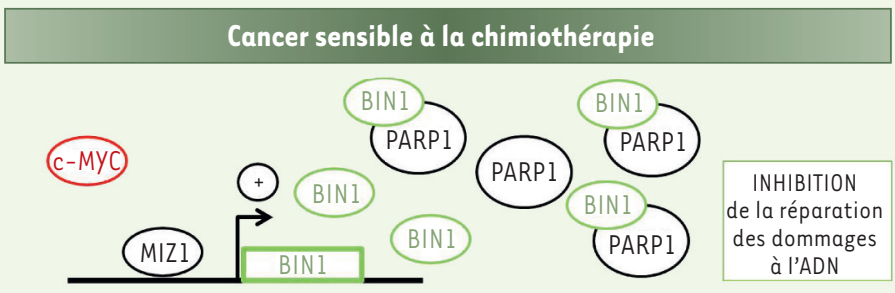

Figure 1. Modèle de régulation de l'expression de BIN1 et de l'activité de PARPI sous la dépendance de $\mathrm{c}-$ MYC. Dans les cancers où C-MYC est abondant, l'interaction de C-MYC avec MIZl bloque I'activation transcriptionnelle de BINI par le biais de MIZI. La diminution conséquente de BINI libère l'activité de PARPI déclenchant alors la résistance des cellules cancéreuses à la chimiothérapie. Dans le cas des cancers sensibles à la chimiothérapie, la faible abondance de C-MYC libère l'activité transcriptionnelle de MIZI causant une augmentation de BINI. L'interaction de BINI et PARPl inhibe la capacité de PARPl à réparer les lésions de l'ADN.

ou BRCA2 est muté ${ }^{\text {[3]. Nous avons }}$ démontré que le domaine BAR (bin amphiphysine rvs) de BIN1 interagit et inhibe le domaine central de PARPI, supprimant ainsi l'activité catalytique de l'enzyme [7] (Figure 2).

Outre son rôle dans la réparation de I'ADN, PARPI est aussi impliquée dans la régulation transcriptionnelle en servant de cofacteur à de multiples facteurs de transcription [9]. Ainsi, l'inhibition de l'activité de PARPl par une interaction directe avec BINl pourrait non seulement réprimer le système de réparation de l'ADN, mais également être impliquée dans l'inhibition de la transactivation des gènes cibles de C-MYC.

\section{c-MYC, MIZ1, BIN1 et PARP1 :}

\section{une boucle de régulation}

Une augmentation du taux de C-MYC peut inhiber l'expression de BIN1. Cette

${ }^{1}$ La mutation de BRCAl entraîne une déficience de la réparation des cassures de l'ADN double-brin en particulier par recombinaison homologue, et de la réparation couplée à la transcription. Des partenaires multiples de BRCAl sont impliqués dans la réparation de l'ADN. réaction antagoniste entre $\mathrm{C}-\mathrm{MYC}$ et $B I N 1$ implique indirectement le facteur de transcription MIZ1 (MYC-interacting zinc finger 1). MIZl se fixe au promoteur de BINl et active sa transcription. Mais un taux élevé de c-MYC réprime l'effet activateur de MIZl sur l'expression de BIN1. Dans ce cas, c-MYC interagit directement avec MIZl, bloquant alors l'activation de la transcription de $B I N I$, et induisant une diminution de la concentration de la protéine. Cette diminution de BINl libère l'activité de PARPI facilitant alors la transactivation des gènes cibles de C-MYC [7]. Cette boucle de régulation met l'accent sur la corrélation entre une forte abondance de c-MYC et le développement d'une chimiorésistance aux traitements qui agissent via la création de dommages à I'ADN [10]. Inversement, une forte quantité de BINl inhibe l'activité de PARPl, causant alors une augmentation de la sensibilité au traitement et une inhibition de l'activité de c-MYC.

Les conséquences des interactions de BINl avec ses partenaires cellulaires dans la transformation cellulaire, les voies de signalisation de réparation de l'ADN, le métabolisme et l'apoptose sont encore mystérieuses. Par exemple, BIN1 interagit avec Ku70 et Ku80, des protéines qui se fixent aux extrémités libres de l'ADN, pour promouvoir la réparation de l'ADN et la stabilité des télomères [11]. Par ailleurs, en réponse à des dommages à l'ADN, la transcription de BINI est activée par le facteur de transcription $\varepsilon 2 \mathrm{Fl}$, et ce mécanisme joue un rôle dans le déclenchement de l'apoptose dépendant d'E2F1 [12].

\section{Implications cliniques}

Le modèle de régulation que nous avons proposé pourrait avoir des implications cliniques. La régulation de PARPI par BINI implique que dans les cancers où prévaut une dérégulation de C-MYC (citons l'exemple du cancer du sein sans altération de BRCAl ou BRCA2), BINI sera présente en faible abondance et PARPI hyperactive. L'interaction entre ces trois acteurs déclenche le mécanisme de résistance des cancers aux drogues qui provoquent des lésions de I'ADN. Cela suggère que l'inhibition de PARP pourrait être un moyen de sensibiliser les cancers présentant une dérégulation de C-MYC. En effet, un essai clinique a dévoilé que l'effet combiné de l'inhibition de PARP à la chimiothérapie classique apporte un bénéfice considérable au traitement des cancers du sein «triple négatifs » (absence des récepteurs des oestrogènes $[\varepsilon R]$, des progestérones $[P R]$ et de l'epidermal growth factor \&GF [HER2]) où c-MYC est systématiquement dérégulé [13]. L'évaluation quantitative ou qualitative de BIN1 pourrait être utilisée comme marqueur biologique prédictif identifiant les cancers qui devraient réellement bénéficier de l'inhibition thérapeutique de PARPI. II serait fort intéressant de déterminer qui, de l'activation de c-MYC ou de la perte de BIN1, serait le meilleur marqueur pour identifier les cancers qui devraient bénéficier de l'ajout de l'inhibiteur chimique de PARP à la 


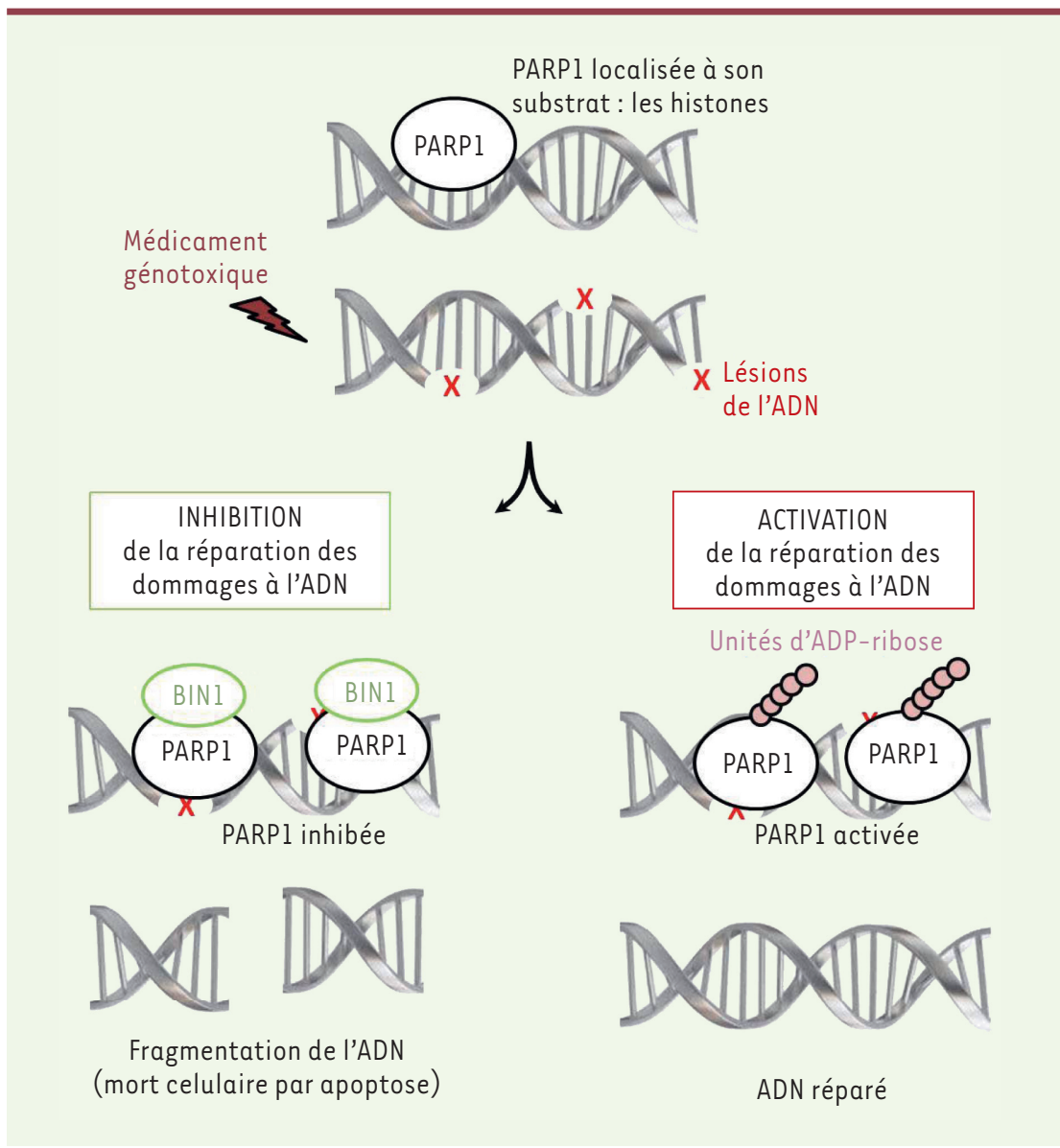

Figure 2. Représentation schématique de la fonction de PARPI après une cure par un médicament génotoxique. Suite à des cassures de l'ADN, PARPl est activée et catalyse la synthèse d'unités d'ADP-ribose au niveau de son domaine d'automodification. Cette modification contribue à la réparation de l'ADN endommagé. L'interaction du domaine BAR de BINI avec le domaine d'automodification de PARPl inhibe l'activation de PARPl. En raison de cette inactivation de PARPl, les dommages à l'ADN persistent, provoquant la mort cellulaire par apoptose.

chimiothérapie classique. Si le modèle que nous proposons est validé sur des tumeurs primaires humaines, de nouvelles approches thérapeutiques visant la chimiorésistance des cancers contrôlés par c-MYC pourraient voir le jour. $\diamond$
C-MYC, PARPI and BIN1 as targets for
therapy of cancer cell resistance

\section{LIENS D'INTÉRÊT}

Les auteurs déclarent n'avoir aucun lien d'intérêt concernant les données publiées dans cet article.

\section{RÉFÉRENCES}

1. Goda K, Bacsó Z, Szabó Z. Multidrug resistance through the spectacle of $\mathrm{P}$-glycoprotein. Curr Cancer Drug Targets $2009 ; 9: 281-97$.

2. Bryant $H \varepsilon$, Schultz $N$, Thomas HD, et al. Specific killing of BRCA2-deficient tumours with inhibitors of poly(ADP-ribose) polymerase. Nature 2005 $434: 913-7$.

3. Helleday T, Bryant HE, Schultz N. Poly(ADP-ribose) polymerase (PARP-1) in homologous recombination and as a target for cancer therapy. Cell Cycle 2005; $4: 1176-8$.

4. Wechsler-Reya R, Sakamuro D, Zhang J, et al. Structural analysis of the human BINI gene. Evidence for tissue-specific transcriptional regulation and alternate RNA splicing. J Biol Chem 1997 ; 272 : 31453-8.

5. Sakamuro D, Elliott KJ, Wechsler-Reya R, Prendergast $\mathrm{GC}$. BINl is a novel MYC-interacting protein with features of a tumour suppressor. Nat Genet 1996 ; $14: 69-77$.

6. Ghaneie A, Zemba-Palko V, Itoh $\mathrm{H}$, et al. Prendergast, Binl attenuation in breast cancer is correlated to nodal metastasis and reduced survival. Cancer Biol Ther $2007 ; 6$ : 192-4.

7. Pyndiah S, Tanida S, Ahmed KM, et al. C-MYC supresses BIN1 to release poly(ADP-ribose) polymerase 1: a mechanism by which cancer cells acquire cisplatin resistance. Sci Signal 2011 ; 4 : ral9.

8. Bryant HE, Schultz N, Thomas HD, et al. Specific killing of BRCA2-deficient tumours with inhibitors of poly(ADP-ribose) polymerase. Nature 2005 ; $434: 913-7$.

9. Ji Y, Tulin AV. The roles of PARPl in gene control and cell differentiation. Curr Opin Genet Dev 2010 ; $20: 512-8$.

10. Sklar MD, Prochownik EV. Modulation of cis-platinum resistance in Friend erythroleukemia cells by c-myc. Cancer Res $1991 ; 51: 2118-23$.

11. Ramalingam A, Farmer GE, Stamato TD, Prendergast GC. Binl interacts with and restrains the DNA endbinding protein complex Ku. Cell Cycle 2007 ; $6: 1914-8$.

12. Cassimere EK, Pyndiah S, Sakamuro D. The c-MYCinteracting proapoptotic tumor suppressor BINl is a transcriptional target for $\varepsilon 2 \mathrm{Fl}$ in response to DNA damage. Cell Death Differ 2009 ; 16 : 1641-53.

13. O’Shaughnessy J, Osborne C, Pippen JE, et al. Iniparib plus chemotherapy in metastatic triple-negative breast cancer. N Engl J Med 2011 ; 364 : 205-14. 